\title{
Nonlinear magneto-optical rotation with frequency-modulated light
}

\author{
D. Budker ${ }^{a, b}$, D. F. Kimball ${ }^{a}$, V. V. Yashchuk ${ }^{a}$, and M. Zolotorev ${ }^{c}$ \\ a Department of Physics, University of California, \\ Berkeley, CA 94720-7300 \\ e-mail: budker@socrates.berkeley.edu \\ $b$ Nuclear Science Division, \\ Lawrence Berkeley National Laboratory, Berkeley CA 94720 \\ c Center for Beam Physics, \\ Lawrence Berkeley National Laboratory, Berkeley CA 94720
}

(Dated: October 25, 2018)

\begin{abstract}
A magnetometric technique is demonstrated that may be suitable for precision measurements of fields ranging from the sub-microgauss level to above the Earth field. It is based on resonant nonlinear magneto-optical rotation caused by atoms contained in a vapor cell with anti-relaxation wall coating. Linearly polarized, frequency-modulated laser light is used for optical pumping and probing. If the time-dependent optical rotation is measured at the first harmonic of the modulation frequency, ultra-narrow $(\sim$ a few $\mathrm{Hz})$ resonances are observed at near-zero magnetic fields, and at fields where the Larmor frequency coincides with half the light modulation frequency. Upon optimization, the sensitivity of the technique is expected to exceed $10^{-11} \mathrm{G} / \sqrt{\mathrm{Hz}}$.
\end{abstract}

When light near-resonant to an atomic transition propagates through an atomic medium immersed in a magnetic field, the light polarization can be affected. For example, when a magnetic field is applied along the direction of light propagation, there is light-power-dependent rotation of the polarization plane known as nonlinear magneto-optical (Faraday) rotation (NMOR). Recently [1], we observed ultra-narrow $(\sim 1 \mathrm{~Hz})$ zero-field resonances in NMOR with rubidium atoms contained in vapor cells with high-quality anti-relaxation coating [2. These resonances arise due to preservation of atomic polarization over thousands of collisions with the walls of the cell. The sensitivity of an NMOR-based magnetometer for sub-microgauss fields could, in principle, exceed $10^{-11} \mathrm{G} / \sqrt{\mathrm{Hz}}$ [3], approaching the fundamental shot-noise limit (given the number of atoms in the cell and the polarization relaxation rate). In the present work, we describe a new technique that involves both inducing and measuring NMOR with a single frequencymodulated light beam. Additional ultra-narrow resonances are observed when the light modulation frequency coincides with twice the Larmor frequency, $2 \Omega_{L}$ (and also with $\Omega_{L}$, etc.), the use of which can extend the dynamic range of an NMOR-based magnetometer to above the Earth field range $(\sim 1 \mathrm{G})$ of interest in many applications.

The high sensitivity of NMOR magnetometry is based in part on the ability of the polarimeter to measure small optical rotation angles. The sensitivity of polarimeters without modulation is limited by low-frequency noise. Therefore, the most successful polarimetric techniques always involve some type of fast modulation. In our previous work [1, 3], we used polarization-modulation polarimetry, where in addition to the vapor cell under investigation, a Faraday rotator is inserted between the crossed polarizer and analyzer. The Faraday rotator modulates the polarization direction at a frequency of $\sim 1 \mathrm{kHz}$, and the rotation angle is determined by measuring the first harmonic of the signal from a photodetector in the dark output of the analyzer. Such a method allows detection of signals at high frequencies, eliminating sensitivity to excess low-frequency noise. However, the polarization modulation technique has a number of shortcomings. Importantly, it is not immune to the problem of "drifting zero" - any relative rotation of the polarizer and analyzer, or a change in the birefringence of optical elements, etc. is detected as a rotation signal indistinguishable from rotation produced by the atoms. In order to avoid such problems, a light frequency modulation technique was introduced in Refs. [国 5 ] and was applied to measurements of linear magneto-optical rotation and parity-violating optical rotation. This technique allowed detection of optical rotation at the light modulation frequency without introducing additional optical elements (such as a Faraday modulator) between the polarizer and analyzer, considerably reducing spurious rotations. Sensitivity to most spurious rotations (including the "drift of zero") is further reduced because such effects generally do not lead to spectral features as sharp as an atomic resonance. Optical pumping with frequency-modulated light was studied and applied to ${ }^{4} \mathrm{He}$ resonance magnetometers [6, 7, 8], but in that work transmission was monitored, in contrast to the measurements of optical rotation performed in the present work. The performance of the ${ }^{4} \mathrm{He}$ magnetometer is limited by laser intensity noise [8], which is not expected to limit the performance of NMOR-based magnetometers.

A simplified diagram of the experimental apparatus is shown in Fig. 1. A paraffin-coated vapor cell (diameter $10 \mathrm{~cm}$ ) containing an isotopically enriched sample of $\mathrm{Rb}$ atoms $\left(\approx 94 \%\right.$ of ${ }^{87} \mathrm{Rb}$; atomic density $\approx 7 \times 10^{9} \mathrm{~cm}^{-3}$ at $20^{\circ} \mathrm{C}$ ) is placed in a multi-layer magnetic shield equipped with a system of magnetic coils within the inner shield that are used to compensate residual magnetic fields 
(to a level of $\sim 0.1 \mu \mathrm{G}$ ), and to apply well-controlled, arbitrarily-directed fields to the cell [1, 3]. The cell is placed between a polarizer and an analyzer oriented at $\approx$ $45^{\circ}$ with respect to each other (a balanced polarimeter). The frequency of the laser (tuned near the D1-line) is modulated with an amplitude typically of a few hundred $\mathrm{MHz}$. For the measurements presented here, we have chosen modulation frequencies up to $\Omega_{m}=2 \pi \times 3 \mathrm{kHz}$ achieved by modulating a piezo actuator $[9]$ in the diode laser (New Focus, Vortex 6000). The amplitudes at the first and second harmonics of $\Omega_{m}$ of the difference signal between the two photodiodes at the outputs of the analyzer are detected with a lock-in amplifier (Stanford Research Systems, SR810DSP). Upon normalization by the time-averaged sum of the photodiode outputs, this represents the signal measured in this experiment (i.e. the effective amplitude of the first or second harmonic of the time-dependent polarization rotation angle).

The signals at the first and second harmonics of $\Omega_{m}$ as a function of longitudinal (along the light propagation direction) magnetic field are shown in Fig. 2. The laser frequency is modulated at $\Omega_{m}=2 \pi \times 1 \mathrm{kHz}$ with modulation amplitude $\Delta \omega=2 \pi \times 220 \mathrm{MHz}$. For the first and the second harmonic signals, the laser is tuned to where the respective signals are of maximal size: the low-frequency slope of the $F=2 \rightarrow F^{\prime}=1$ resonance $\left(F, F^{\prime}\right.$ are the total angular momenta of the lower and upper state) for the first harmonic (Fig. 因(a)), and to the center of the $F=2 \rightarrow F^{\prime}=1$ resonance for the second harmonic.

For both harmonics, a narrow, dispersively-shaped resonance is seen in Fig. 2 near $B=0$. Its origin is similar to that of the ultra-narrow resonances observed in the previous work [1: the atoms are pumped by linearlypolarized light into a state aligned [10] along the light polarization; optically-pumped atomic alignment evolves due to the applied magnetic field; the resulting atomic polarization [11 is "probed" by the light, leading to a modified light polarization at the output of the cell. For the zero-field resonances in Fig. 2, the modulation frequency is much faster than the Larmor frequency and the optical pumping rate for the cell, so that frequency modulation is equivalent to spectral broadening of the pumping light, which does not significantly affect the pumping process. On the other hand, modulation is essential at the probing stage. As the laser frequency is scanned through the atomic resonance, there is a time-dependent optical rotation, so the signal contains various harmonics of $\Omega_{m}$. The peaks in the magnetic field dependence of the signal occur at $\Omega_{L} \equiv g_{F} \mu B= \pm \gamma_{\mathrm{rel}} / 2$, where $g_{F}$ is the Landé factor $\left(g_{F}=1 / 2\right.$ for the $F=2$ state of $\left.{ }^{87} \mathrm{Rb}\right)$, $\mu \approx 2 \pi \cdot 1.40 \mathrm{MHz} / \mathrm{G}$ is the Bohr magneton, and $\gamma_{\mathrm{rel}}$ $(\sim 2 \pi \cdot 1 \mathrm{~Hz}$ for the data in Fig. 2) is the relaxation rate of atomic polarization. The zero-field resonances can be applied to low-field NMOR-magnetometry [3], with all of the advantages of the frequency-modulation technique as in the case of linear optical rotation 四司.
Additional resonances are seen at magnetic field magnitudes of $714.4 \mu \mathrm{G}\left(\Omega_{m}=2 \Omega_{L}\right)$ and $1428.9 \mu \mathrm{G}\left(\Omega_{m}=\right.$ $\Omega_{L}$ ) for the first and second harmonics, respectively (Fig. 2). For these resonances, there are both dispersivelyshaped in-phase signals (Fig. 2(a,c)) and $\pi / 2$ out of phase (quadrature) components peaked at the centers of these resonances (Fig. 2(b,d)). The origin of the resonances can be understood as follows (Fig. 3). As the laser frequency is modulated, the optical pumping rate changes depending on the instantaneous detuning of the laser from the atomic transition. This occurs with periodicity dictated by the modulation frequency $\Omega_{m}$. On the other hand, the state of atomic polarization also changes periodically due to the presence of the magnetic field. In the case of alignment transverse to the magnetic field, this change occurs at frequency $2 \Omega_{L}$ (alignment precesses at $\Omega_{L}$, but it returns to the same state after a half of the corresponding period). If the optical pumping rate is synchronized with Larmor precession, a resonance occurs, and the atomic medium is optically pumped into an aligned state whose axis rotates at $\Omega_{L}$. The optical properties of the medium are thus being modulated at $2 \Omega_{L}$, causing rotation of the light polarization at this frequency. This leads to dispersively-shaped resonances in optical rotation that are detected as the signal at the appropriate harmonic of $\Omega_{m}$. The quadrature components (Fig. 2(b,d)) arise because exactly on resonance, the aligned atoms produce maximum optical rotation when the alignment axis is at an angle of $\pi / 4$ to the direction of the light polarization. (There is no quadrature component at zero magnetic field because atomic alignment does not rotate at all in this case.) Since the widths of the additional resonances at $\Omega_{m}=2 \Omega_{L}$ and $\Omega_{m}=\Omega_{L}$ are determined by $\gamma_{\text {rel }}$ as for the zero-field resonance, they are similarly narrow. In addition to these resonances, there are also smaller resonances in the first- and secondharmonic signals, generally occurring for commensurate $\Omega_{m}$ and $\Omega_{L}$. There are several mechanisms that lead to these resonances, which will be described in detail elsewhere.

The overall slope of the curves in Fig. 2 is due to the socalled transit effect (see e.g. [1] and references therein), for which the peaks occur at $B= \pm \gamma_{t r} / 2 g_{F} \mu \sim 50 \mathrm{mG}$, where $\gamma_{t r}$ is the rate of atoms' transit through the laser beam.

The spectral dependences of various signals are shown in Fig. 1. Fig. 4 (a) depicts the spectrum of the first harmonic signal obtained when the magnetic field was fixed at $2.2 \mu \mathrm{G}$ (corresponding to maximum signal), and the central frequency of the laser was scanned across the hyperfine structure of the Rb D1-line (the transmission spectrum is shown for comparison in Fig. 四(d)). Comparing this spectrum with the NMOR spectrum obtained without frequency modulation (as in earlier work [1, 3]) shown in Fig. 1(c), it is seen that the spectral profile of the signal with frequency modulation is similar 
to $d \varphi(\nu) / d \nu$, where $\varphi(\nu)$ is the NMOR angle and $\nu$ is the laser frequency. Fig. A(b) shows the spectrum obtained with the magnetic field of $B=(714.4+2.2) \mu \mathrm{G}$, corresponding to an $\Omega_{m}=2 \Omega_{L}$ resonance (Fig. 2). The spectral dependence of the quadrature component of the first harmonic for $B=714.4 \mu \mathrm{G}$ (not shown) is similar to that of the in-phase component. The signals acquired at the second harmonic of $\Omega_{m}$ (not shown) have spectra resembling $d^{2} \varphi(\nu) / d \nu^{2}$.

We have investigated the dependence of the signals on the modulation amplitude $\Delta \omega$ and on the light power, and found similar behavior for the zero-field and the $\Omega_{m}=2 \Omega_{L}$ resonances. The maximum first harmonic amplitude is achieved for modulation amplitude $\Delta \omega$ comparable to the Doppler width of the transition $\sim 300 \mathrm{MHz}$. The signals are proportional to light power at low powers, peak at around $30 \mu \mathrm{W}$, and decrease with further power increase due to light broadening. Subsequent work will include systematic optimization of all parameters for highest magnetometric sensitivity, as it was done earlier [3] for low-field NMOR with conventional polarizationmodulation polarimetry. Additional improvement may be expected if the pump and probe laser beams are separated, and the modulation method is optimized (e.g., a combination of frequency and amplitude modulation). With the present unoptimized parameters and set-up, we estimate that the shot-noise-limited sensitivity of the device is $\sim 10^{-11} \mathrm{G} / \sqrt{\mathrm{Hz}}$ (comparable or superior, e.g., to the most sensitive superconducting quantum interference (SQUID) sensors). Also note that shot-noise-limited operation in the Earth-field range will be facilitated by the reduced laser noise at high frequencies, and by the fact that this method uses resonant optical rotation which allows suppression of the common mode noise of the two photodetectors and spurious rotations. Due to the absence of inertia in magnetic spin-precession, a magnetometer based on this technique (which employs transverse polarization) could, in principle, also have a high band width ultimately limited by electronics.

In conclusion, we have demonstrated a novel magnetometric technique based on nonlinear optical rotation with frequency-modulated light. This technique is useful for near zero-field magnetometry and it also opens a possibility for ultra-sensitive magnetometers with broad dynamic range that includes the Earth field range, of importance to many applications, such as geophysics, magnetic prospecting and navigation. Possible new applications also include nuclear magnetic resonance spectroscopy and imaging using weakly-polarized samples that would not require any additional magnet, except the Earth itself [12]. Narrow magneto-optical resonances may also find applications in atomic tests of fundamental symmetries [13]. For example, it may be advantageous to carry out a search for a parity- and time-reversal invariance violating electric dipole moment of $\mathrm{Cs}$ in the presence of a $\mathrm{Rb}$ co-magnetometer in a bias magnetic field. Due to the difference between the Landé factors for $\mathrm{Rb}$ and $\mathrm{Cs}$, this avoids "locking" of their polarizations to each other. In addition, presence of a bias field eliminates the effects of stray transverse fields.

The authors are grateful to E. B. Alexandrov for stimulating discussions. This work is supported by the Office of Naval Research (grant N00014-97-1-0214). D. B. also wishes to acknowledge support by an NSF CAREER grant.

[1] D. Budker, V. Yashchuk, and M. Zolotorev, Phys. Rev. Lett. 81(26), 5788 (1998).

[2] E. B. Alexandrov, M. V. Balabas, A. S. Pasgalev, A. K. Vershovskii, and N. N. Yakobson, Laser Phys. (Russia) p. 244 (1996).

[3] D. Budker, D. F. Kimball, S. M. Rochester, V. V. Yashchuk, and M. Zolotorev, Phys. Rev. A 62(4), 043403 (2000).

[4] L. M. Barkov and M. S. Zolotorev, Pis'ma v ZhETF 28(8), 544 (1978), (JETP Lett. 28(8), 503 (1978).).

[5] L. M. Barkov, M. Zolotorev, and D. Melik-Pashaev, Pis'ma v ZhETF, 48(3), 134 (1988), (JETP Lett. 48(3), 144 (1988).).

[6] B. Cheron, H. Gilles, J. Hamel, O. Moreau, and E. Noel, Opt. Commun. 115(1-2), 71 (1995).

[7] B. Cheron, H. Gilles, J. Hamel, O. Moreau, and E. Noel, J. Phys. II (France) 6(2), 175 (1996).

[8] H. Gilles, J. Hamel, and B. Cheron, Rev. Sci. Instrum. 72(5), 2253 (2001).

[9] We have also carried out measurements where laser frequency was modulated by changing the diode laser current at frequencies up to $\sim 100 \mathrm{kHz}$. (Modulation at frequencies up to $100 \mathrm{MHz}$ is possible with this laser.) At low modulation frequencies, results for both type of laser modulation are similar, although for a quantitative analysis, it is necessary to take into account nonnegligible light amplitude modulation which accompanies frequency modulation when current modulation is used.

[10] Alignment is a particular case of atomic polarization corresponding to an anisotropy characterized by a preferred axis, rather than by a preferred direction as in the case of orientation. Alignment is represented by a rank-two (quadrupole) irreducible tensor component of the density matrix, while orientation is represented by a rank-one component.

[11] The resulting polarization is alignment rotated due to Larmor precession at low light powers, and a more complicated atomic polarization state when optical pumping is saturated (see D. Budker, D. F. Kimball, S. M. Rochester, and V. V. Yashchuk, Phys. Rev. Lett. 85(10), 2088 (2000).).

[12] A. H. Trabesinger, R. McDermott, M. Muck, E. L. Hahn, J. Clarke, and A. Pines, in 42-nd Experimental NMR Conference, Orlando, FL, USA; March Meeting of the APS, Seattle, WA, USA (2001), in Books of Abstracts.

[13] V. Yashchuk, D. Budker, and M. Zolotorev (1999), p. 177, Trapped Charged Particles and Fundamental Physics Asilomar, CA, USA 31 Aug.-4 Sept. 1998 AIP Conf. Proc. \#457. 


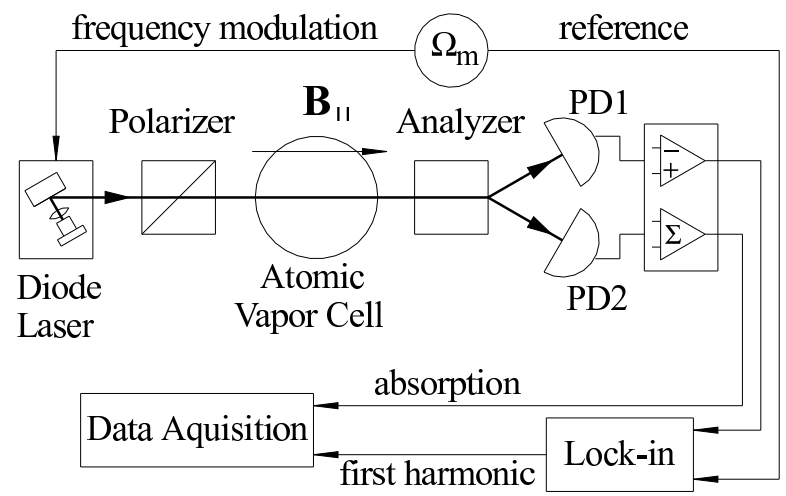

FIG. 1: Simplified schematic of the apparatus.

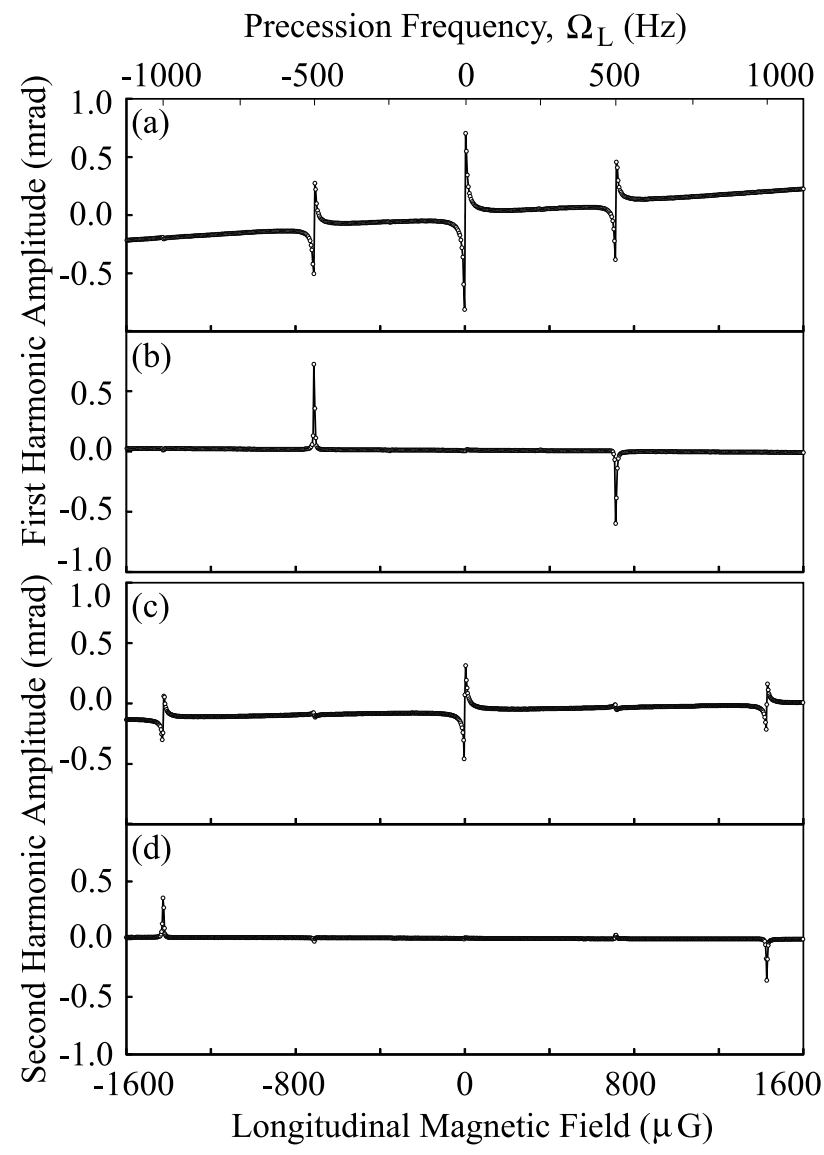

FIG. 2: Signals detected at the first harmonic (a,b) and second harmonic $(\mathrm{c}, \mathrm{d})$ of $\Omega_{m}$ as a function of longitudinal magnetic field. The laser power was $15 \mu \mathrm{W}$, beam diameter $\sim 2 \mathrm{~mm}, \Omega_{m}=2 \pi \times 1 \mathrm{kHz}, \Delta \omega=2 \pi \times 220 \mathrm{MHz}$. Traces $(\mathrm{a}, \mathrm{c})$ and $(\mathrm{b}, \mathrm{d})$ correspond to the in-phase and the quadrature outputs of the signals from the lock-in detector, respectively. 
(a) Atomic Alignment Pumping and Precession $\left(\Omega_{\mathrm{L}}\right)$

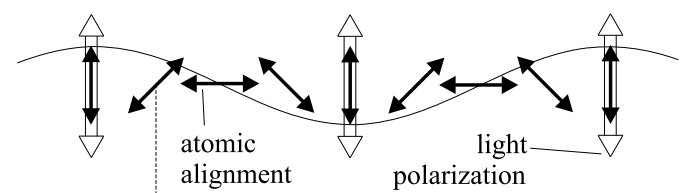

(b) Probe Optical Rotation $\left(2 \Omega_{\mathrm{L}}\right)$

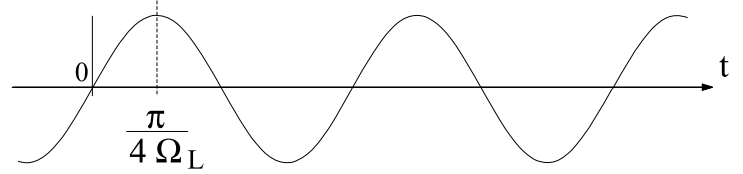

FIG. 3: Origin of the NMOR resonances at $\Omega_{m}=2 \Omega_{L}, \Omega_{L}$. (a) Atomic alignment precesses with Larmor frequency (line). Linearly-polarized light periodically interacts with the atoms. (b) Optical rotation of an unmodulated probe beam occurs at frequency $2 \Omega_{L}$.

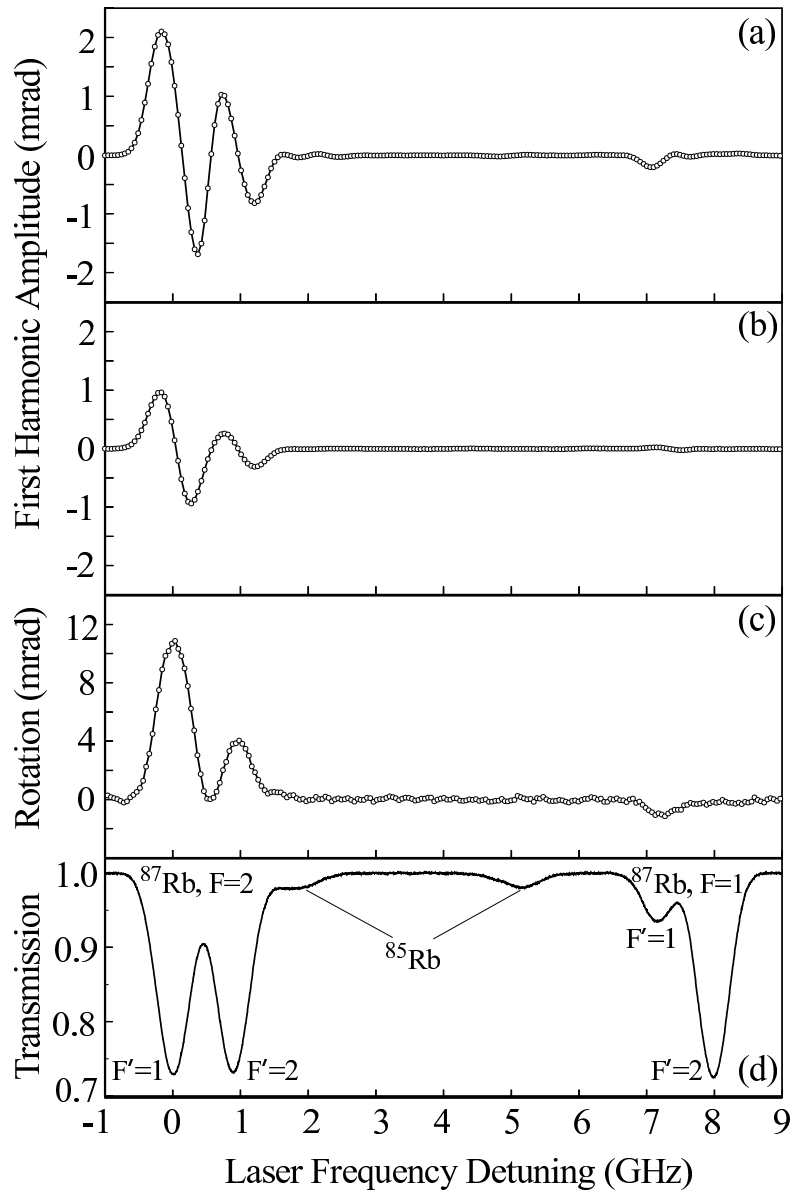

FIG. 4: Spectral dependences of various signals. (a) First harmonic amplitude measured at $B=2.2 \mu \mathrm{G}$ (the narrow zero-field resonance), light power $\sim 15 \mu \mathrm{W}$; (b) same at $B=$ $714.4+2.2 \mu \mathrm{G}$ (the $\Omega_{m}=2 \Omega_{L}$ resonance), same light power, $\Omega_{m}=2 \pi \times 1 \mathrm{kHz}, \Delta \omega=2 \pi \times 220 \mathrm{MHz}$; (c) nonlinear Faraday rotation recorded without frequency modulation (as in 11 , 3 ), $B=2.2 \mu \mathrm{G}$, same light power; (d) transmission through the vapor cell for low light power $(0.4 \mu \mathrm{W})$. For all traces, light beam diameter $\sim 2 \mathrm{~mm}$. 